\title{
FORCING AND THE HALPERN-LÄUCHLI THEOREM
}

\author{
NATASHA DOBRINEN AND DANIEL HATHAWAY
}

\begin{abstract}
We investigate the effects of various forcings on several forms of the Halpern-Läuchli Theorem. For inaccessible $\kappa$, we show they are preserved by forcings of size less than $\kappa$. Combining this with work of Zhang in [17] yields that the polarized partition relations associated with finite products of the $\kappa$-rationals are preserved by all forcings of size less than $\kappa$ over models satisfying the Halpern-Läuchli Theorem at $\kappa$. We also show that the HalpernLäuchli Theorem is preserved by $<\kappa$-closed forcings assuming $\kappa$ is measurable, following some observed reflection properties.
\end{abstract}

\section{INTRODUCTION}

The Halpern-Läuchli Theorem [7] is a Ramsey theorem for products of finitely many trees of height $\omega$ which are finitely branching and have no terminal nodes. It was discovered as a central lemma to the proof in [8] that the Boolean Prime Ideal Theorem (the fact that every ideal in a Boolean algebra can be extended to a prime ideal) is strictly weaker than the Axiom of Choice, over Zermelo-Fraenkel set theory. Many variations have been studied, some of which are equivalent to the statement that the BPI is strictly weaker than the Axiom of Choice. Recent compendia of various versions of the Halpern-Läuchli Theorem appear in [15] and [3]. The Halpern-Läuchli Theorem has found numerous applications in proofs of partition relations for countable structures, directly to products of rationals (see [11]) and via the closely related theorem of Milliken for strong trees [12] to finite sets of rationals (see [1]) and the Rado graph (see [13] and [10]).

Some years after the Halpern-Läuchli Theorem was discovered, Harrington found a proof which uses the techniques and language of forcing, though without actually passing to a generic extension of the ground model. Though this proof was well-known in certain circles, a published version did not appear until [16].

2010 Mathematics Subject Classification. Fill in.

The first author was partially supported from National Science Foundation Grant DMS-1600781. 
Shelah applied this proof method of Harrington in [14] to prove a partition theorem (analogue of Milliken's Theorem) for trees on $2^{<\kappa}$, where $\kappa$ is a cardinal whose measurability is preserved by adding many Cohen subsets of $\kappa$. This result was extended and applied by Džamonja, Larson and Mitchell in [4] and [5] to obtain partition relations on the $\kappa$-rationals and $\kappa$-Rado graph. This work, as well as the exposition in Chapter 3 of Todorcevic's book [15] informed the authors' previous work on variations of the Halpern-Läuchli Theorem for more than one tree at uncountable cardinals. In [2], we mapped out the implications between weaker and stronger forms of the Halpern-Läuchli Theorem on trees of uncountable height, and found a better upper bound for the consistency strength of the theorem holding for finitely many trees at a measurable cardinal. Building on work in [4] and [2], Zhang proved a stronger tail-cone version at measurable cardinals and applied it to obtain the analogue of Laver's partition relation for products of finitely many trees on a measurable cardinal (see [17]).

It is intriguing that all theorems for trees of uncountable height proved so far have required assumptions beyond ZFC. In fact, it is still unknown whether such partition relation theorems for trees at uncountable heights simply are true in ZFC or whether they entail some large cardinal strength. For more discussion of this main problem and other related open problems, see Section 7.

In this paper, we are interested in which forcings preserve the HalpernLäuchli Theorem for trees of uncountable height, once it is known to hold. Section 2 contains basic definitions, most of which are found in [2] and [17. It also contains an equivalence between the tail-cone version of the Halpern-Läuchli Theorem and a modified version which is easier to satisfy in practice. Section 3 contains a new method which constructs a tree in the ground model using forcing names; this is called the derived tree from a name for a tree. The Derived Tree Theorem is proved there.

The Derived Tree Theorem is applied in Section 4 to show that small forcings preserve the Halpern-Läuchli Theorem and its tail-cone version. As the partition relation on finite products of $\kappa$-rationals holds in any model where the tail-cone version holds (by work of Zhang in [17]), our work shows that this partition relation is preserved by any further small forcing.

Section 5 presents some instances when the somewhere dense version (SDHL) has reflection properties. Thus, if SDHL holds for a stationary set of cardinals below a strongly inaccessible cardinal $\kappa$, then it holds at $\kappa$. Second, we prove that for a measurable cardinal $\kappa$, SDHL holds at $\kappa$ if and only if the set of cardinals below $\kappa$ where SDHL holds is a 
member of each normal ultrafilter on $\kappa$. We apply this to show that $<\kappa$-closed forcings preserve SDHL at measurable cardinals.

Finally, in Section 6, we provide a model of ZFC where SDHL holds at some regular cardinal which is not weakly compact. This produces a different model than the one in [17], one that is obtained by a large collection of forcings. Section 7 contains several key questions brought to the fore by this work. Although interesting in their own right, they are all sub-problems of the main open problem: Is the Halpern-Läuchli Theorem for trees of any cardinal height simply true in ZFC?

\section{BAsiC Definitions}

We review here some fundamental definitions from [2]. Given sequences $s$ and $t$, the notation $s \sqsubseteq t$ means that $s$ is an initial segment of $t$; the notation $s \sqsubset t$ denotes that $s$ is a proper initial segment of $t$. A set $T \subseteq{ }^{<\kappa} \kappa$ is a tree iff it is closed under taking initial segments. For $t \in^{<\kappa} \kappa$, let $\operatorname{Dom}(t)$ denote the domain of $t$. We shall also call this the length of $t$. Given $\alpha \leq \operatorname{Dom}(t)$, we write $t \uparrow \alpha$ for the unique initial segment of $t$ of length $\alpha$.

Definition 2.1. A tree $T$ is a regular $\kappa$-tree if $T \subseteq{ }^{<\kappa} \kappa$ and

(1) $T$ is a $\kappa$-tree; that is, $T$ has height $\kappa$ and each level of $T$ has size $<\kappa$

(2) Every maximal branch of $T$ has length $\kappa$;

(3) $T$ is perfect, meaning that for any $t \in T$, there are incomparable $s, u \sqsupseteq t$ in $T$.

Note that if $\kappa$ is a regular cardinal and there exists a regular $\kappa$-tree, then $\kappa$ must be a strongly inaccessible cardinal. However, there do exist regular $\kappa$-trees for singular cardinals $\kappa$. Specifically, there exists a regular $\kappa$-tree if $2^{\lambda}<\kappa$ for all $\lambda<\operatorname{cf}(\kappa)$.

Given a set $X \subseteq{ }^{<\kappa} \kappa$ and an ordinal $\alpha<\kappa$, let $X(\alpha)$ denote the set of sequences in $X$ of length $\alpha$; that is,

$$
X(\alpha)=\{t \in X: \operatorname{Dom}(t)=\alpha\} .
$$

Given sets $X, Y \subseteq{ }^{<\kappa} \kappa$, we say that $X$ dominates $Y$ if to each $y \in Y$ there corresponds at least one $x \in X$ such that $x \sqsupseteq y$. Given $t \in{ }^{<\kappa} \kappa$, Cone $(t)$ is the set of all $t^{\prime} \sqsupseteq t$ in ${ }^{<\kappa} \kappa$.

Definition 2.2. Given $1 \leq d<\omega$ and a sequence $\left\langle X_{i} \subseteq{ }^{<\kappa} \kappa: i<d\right\rangle$, define the level product of the $X_{i}$ 's to be

$$
\bigotimes_{i<d} X_{i}:=\left\{\left\langle x_{i}: i<d\right\rangle:(\exists \alpha<\kappa)(\forall i<d) x_{i} \in X_{i}(\alpha)\right\} .
$$


Definition 2.3. Let $1 \leq d<\omega$. Given $\kappa$-trees $T_{0}, \ldots, T_{d-1}$, we call a sequence $\left\langle X_{i}: i<d\right\rangle$ a somewhere dense level matrix if there are ordinals $\alpha<\beta<\kappa$ and a sequence $\left\langle t_{i} \in T_{i}(\alpha): i<d\right\rangle$ such that each $X_{i}$ is a subset of $T_{i}(\beta)$, and further, $X_{i}$ dominates $T_{i}(\alpha+1) \cap$ Cone $\left(t_{i}\right)$.

The following is the somewhere dense version of the Halpern-Läuchli Theorem, which we denote by $\operatorname{SDHL}(d, \sigma, \kappa)$. Given a coloring $c$ and a set $S$, we say $c$ is monochromatic on $S$ if and only if $|c " S|=1$.

Definition 2.4. For $1 \leq d<\omega$ and cardinals $0<\sigma<\kappa$ with $\kappa$ infinite, $\operatorname{SDHL}(d, \sigma, \kappa)$ is the statement that given any sequence $\left\langle T_{i}: i<d\right\rangle$ of regular $\kappa$-trees and any coloring

$$
c: \bigotimes_{i<d} T_{i} \rightarrow \sigma
$$

there exists a somewhere dense level matrix $\left\langle X_{i} \subseteq T_{i}: i<d\right\rangle$, such that $c$ is monochromatic on $\bigotimes_{i<d} X_{i}$.

When we say $\operatorname{SDHL}(d, \sigma, \kappa)$ is true, this implies that $\operatorname{SDHL}(d, \sigma, \kappa)$ is defined, so $1 \leq d<\omega$ and $\sigma<\kappa$. However, $\operatorname{SDHL}(d, \sigma, \kappa)$ does not imply $\kappa$ is an inaccessible cardinal. If $1 \leq d<\omega$ and $0<\sigma<\kappa$ but there are no regular $\kappa$-trees, then $\operatorname{SDHL}(d, \sigma, \kappa)$ is vacuously true. This convention makes Proposition 5.1 more convenient.

Often one wants to apply SDHL, but restricted to certain levels. This is readily seen to be possible for regular cardinals.

Fact 2.5. Let $1 \leq d<\omega, \kappa$ a regular cardinal, and $0<\sigma<\kappa$ be given, and assume $\operatorname{SDHL}(d, \sigma, \kappa)$ holds. Let $T_{i}(i<d)$ be a sequence of regular $\kappa$-trees (so $\kappa$ is strongly inaccessible). Let $c: \bigotimes_{i<d} T_{i} \rightarrow \sigma$ be a coloring, and let $A \subseteq \kappa$ be cofinal in $\kappa$. Then there exist $\alpha<\beta<\kappa$ both in $A,\left\langle t_{i} \in T_{i}(\alpha): i<d\right\rangle$ and $\left\langle X_{i} \subseteq T_{i}(\beta): i<d\right\rangle$ such that each $X_{i}$ dominates $T_{i}(\alpha+1) \cap$ Cone $\left(t_{i}\right)$ and $c$ is monochromatic on $\bigotimes_{i<d} X_{i}$.

The other two forms of the Halpern-Läuchli Theorem we will consider involve the notion of a strong subtree. In this paper, by successor we mean immediate successor.

Definition 2.6. Let $T$ be a regular $\kappa$-tree. A tree $T^{\prime} \subseteq T$ is a strong subtree of $T$ as witnessed by some set $A \subseteq \kappa$ cofinal in $\kappa$ if $T^{\prime}$ is regular and for each $t \in T^{\prime}(\alpha)$ for $\alpha<\kappa$,

1) $\alpha \in A$ implies every successor of $t$ in $T$ is also in $T^{\prime}$;

2) $\alpha \notin A$ implies that $t$ has a unique successor in $T^{\prime}$ on level $\alpha+1$. We refer to an ordinal $\alpha \in A$ as a splitting level of $T^{\prime}$.

The following is the strong tree version of the Halpern-Läuchli Theorem, which we denote by $\operatorname{HL}(d, \sigma, \kappa)$. 
Definition 2.7. For $1 \leq d<\omega$ and cardinals $0<\sigma<\kappa$ with $\kappa$ infinite, $\operatorname{HL}(d, \sigma, \kappa)$ is the following statement: Given any sequence $\left\langle T_{i}: i<d\right\rangle$ of regular $\kappa$-trees and a coloring $c: \bigotimes_{i<d} T_{i} \rightarrow \sigma$, there exists a sequence of trees $\left\langle T_{i}^{\prime}: i<d\right\rangle$ such that the following hold:

(1) Each $T_{i}^{\prime}$ is a strong subtree of $T_{i}$ as witnessed by the same set $A \subseteq \kappa$, independent of $i$; and

(2) $c$ is monochromatic on $\bigcup_{\alpha \in A} \bigotimes_{i<d} T_{i}^{\prime}(\alpha)$.

Just as in Fact 2.5. HL can be applied but restricted to any $A \subseteq \kappa$ cofinal in $\kappa$. Specifically, if $\kappa$ is a regular cardinal, and we have a sequence of regular $\kappa$-trees $T_{i}$ for $i<d$, then given any $A \subseteq \kappa$ cofinal in $\kappa$, there is a sequence of strong subtrees $T_{i}^{\prime} \subseteq T_{i}$ for $i<d$ all witnessed by the same set of splitting levels $B \subseteq A$ and $c$ is monochromatic on $\bigcup_{\beta \in B} \bigotimes_{i<d} T_{i}^{\prime}(\beta)$. In practice, one usually uses regular $\kappa$-trees $T_{i}$ such that for each $\alpha \in A$, each node of length $\alpha$ in $T_{i}$ has two or more successors.

Although in [2] we stated that $\operatorname{HL}(d, \sigma, \kappa)$ is equivalent to $\operatorname{SDHL}(d, \sigma, \kappa)$ for any weakly compact $\kappa$, our proof never actually used the assumption that $\kappa$ was weakly compact, only that is was strongly inaccessible.

Proposition 2.8. $H L(d, \sigma, \kappa)$ is equivalent to $\operatorname{SDHL}(d, \sigma, \kappa)$ for all inaccessible $\kappa$.

Finally, the following is the tail cone version of the Halpern-Läuchli Theorem, which we shall denote by $\operatorname{HL}^{t c}(d,<\kappa, \kappa)$, which appears in Section 3 of [17].

Definition 2.9. For $1 \leq d<\omega$ and $\kappa$ an infinite cardinal, $\mathrm{HL}^{t c}(d,<$ $\kappa, \kappa)$ is the following statement: Given a sequence of regular $\kappa$-trees $\left\langle T_{i}: i<d\right\rangle$, a sequence of nonzero cardinals $\left\langle\sigma_{\zeta}<\kappa: \zeta<\kappa\right\rangle$, and a sequence of colorings $c_{\zeta}: \bigotimes_{i<d} T_{i} \rightarrow \sigma_{\zeta}$ for $\zeta<\kappa$, there exists a sequence of trees $\left\langle T_{i}^{\prime}: i<d\right\rangle$ such that

(1) Each $T_{i}^{\prime}$ is a strong subtree of $T_{i}$ as witnessed by the same set $A \subseteq \kappa$, independent of $i$; and

letting $\left\{\alpha_{\zeta}: \zeta<\kappa\right\}$ denote the increasing enumeration of $A$,

(2) For each pair of ordinals $\zeta \leq \xi<\kappa$, given any sequence $\left\langle t_{i} \in\right.$ $\left.T_{i}^{\prime}\left(\alpha_{\xi}\right): i<d\right\rangle$, we have

$$
c_{\zeta}\left\langle t_{i}: i<d\right\rangle=c_{\zeta}\left\langle t_{i}\left\lceil\alpha_{\zeta}: i<d\right\rangle .\right.
$$

In other words, the $c_{\zeta}$-color of a tuple $\vec{t}=\left\langle t_{i}: i<d\right\rangle$ on the $\xi$-th splitting level (for $\xi \geq \zeta$ ) is the same as the $c_{\zeta}$-color of $\vec{t}$ restricted to the $\zeta$-th splitting level. 
Note that $\operatorname{SDHL}(d, \sigma, \kappa), \operatorname{HL}(d, \sigma, \kappa)$, and $\operatorname{HL}^{t c}(d, \sigma, \kappa)$ are all statements about $V_{\kappa+1}$. We will need the following concept later.

Definition 2.10. For $1 \leq d<\omega$ and $\kappa$ an infinite cardinal, the modified $\mathrm{HL}^{t c}(d,<\kappa, \kappa)$ is just $\operatorname{HL}^{t c}(d,<\kappa, \kappa)$ but with (2) replaced with the following:

$\left(2^{*}\right)$ There is a function $\mu: \kappa \rightarrow \kappa$ such that $(\forall \gamma<\kappa) \mu(\gamma) \geq \gamma$ satisfying the following: For each pair of ordinals $\zeta \leq \gamma<\kappa$, given any sequence $\left\langle t_{i} \in T_{i}^{\prime}\left(\alpha_{\gamma}\right): i<d\right\rangle$, we have

$$
c_{\mu(\zeta)}\left\langle t_{i}: i<d\right\rangle=c_{\mu(\zeta)}\left\langle t_{i}\left\lceil\alpha_{\zeta}: i<d\right\rangle .\right.
$$

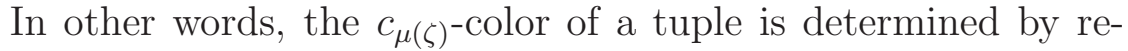
stricting to the $\zeta$-th splitting level.

Proposition 2.11. Fix $1 \leq d<\omega$ and $\kappa$ a strongly inaccessible cardinal. Then $H L^{t c}(d,<\kappa, \kappa)$ and its modified version are equivalent.

Proof. It is clear that the unmodified version implies the modified version holds: Just set $\mu: \kappa \rightarrow \kappa$ to be the identity function. For the other direction, assume the modified version holds.

Let $\left\langle T_{i}: i<d\right\rangle$ be a sequence of regular $\kappa$-trees and $\left\langle c_{\zeta}: \zeta<\kappa\right\rangle$ a sequence of colorings, where $c_{\zeta}: \bigotimes_{i<d} T_{i} \rightarrow \sigma_{\zeta}$ for each $\zeta<\kappa$. We will find strong subtrees $T_{i}^{\prime} \subseteq T_{i}$ for $i<d$ all witnessed by the same set of splitting levels $A=\left\{\alpha_{\gamma}: \gamma<\kappa\right\}$ such that (2) of the definition of $\mathrm{HL}^{t c}$ holds.

For each $\gamma<\kappa$, let $\sigma_{\gamma}^{\prime}$ be the product of the cardinals $\sigma_{\zeta}$ for $\zeta \leq \gamma$. Since $\kappa$ is strongly inaccessible, each $\sigma_{\gamma}^{\prime}$ is strictly less than $\kappa$. For each $\gamma<\kappa$, let $c_{\gamma}^{\prime}: \bigotimes_{i<d} T_{i} \rightarrow \sigma_{\gamma}^{\prime}$ be a coloring which encodes the colorings $c_{\zeta}$ for $\zeta \leq \gamma$. That is, given $\gamma<\kappa$ and $\vec{t} \in \bigotimes_{i<d} T_{i}$, the sequence $\left\langle c_{\zeta}(\vec{t}): \zeta \leq \gamma\right\rangle$ can be recovered from knowing $c_{\gamma}^{\prime}(\vec{t})$. Thus, given any $\vec{s}, \vec{t}$ with the same $c_{\gamma}^{\prime}$-color, then $\vec{s}, \vec{t}$ have the same $c_{\zeta}$-color for all $\zeta \leq \gamma$.

Now apply the modified version to the trees $\left\langle T_{i}: i<d\right\rangle$ and the colorings $\left\langle c_{\gamma}^{\prime}: \gamma<\kappa\right\rangle$ to produce strong subtrees $T_{i}^{\prime} \subseteq T_{i}$, each with the same set of splitting levels $A=\left\{\alpha_{\gamma}: \gamma<\kappa\right\}$, and some fixed function $\mu: \kappa \rightarrow \kappa$ such that for any pair of ordinals $\zeta \leq \gamma<\kappa$, the $c_{\mu(\zeta)}^{\prime}$-color of a $d$-tuple on the splitting level $\alpha_{\gamma}$ is determined by restricting to the splitting level $\alpha_{\zeta}$. Then for any pair of ordinals $\zeta \leq \gamma<\kappa$ and any $\left\langle t_{i} \in T_{i}^{\prime}\left(\alpha_{\gamma}\right): i<d\right\rangle$,

$$
c_{\mu(\zeta)}^{\prime}\left\langle t_{i}: i<d\right\rangle=c_{\mu(\zeta)}^{\prime}\left\langle t_{i} \uparrow \alpha_{\zeta}: i<d\right\rangle .
$$

Recalling that $c_{\mu(\zeta)}^{\prime}$ encodes the colorings $c_{\psi}$ for all $\psi \leq \mu(\zeta)$ and that the function $\mu$ satisfies $\mu(\gamma) \geq \gamma$ for each $\gamma<\kappa$, it follows that for each 
pair of ordinals $\zeta \leq \gamma<\kappa$ and any $\left\langle t_{i} \in T_{i}^{\prime}\left(\alpha_{\gamma}\right): i<d\right\rangle$,

$$
c_{\zeta}\left\langle t_{i}: i<d\right\rangle=c_{\zeta}\left\langle t_{i} \uparrow \alpha_{\zeta}: i<d\right\rangle \text {. }
$$

This is precisely (2) of the definition of $\mathrm{HL}^{t c}$.

Observation 2.12. $\mathrm{HL}^{t c}(d,<\kappa, \kappa)$ implies $(\forall \sigma<\kappa) \mathrm{HL}(d, \sigma, \kappa)$. This can be seen by using a sequence of colorings $\left\langle c_{\zeta}: \zeta<\kappa\right\rangle$ that are all constant except the first one $c_{0}$. Applying $\mathrm{HL}^{t c}(d,<\kappa, \kappa)$ produces strong subtrees $T_{i}^{\prime} \subseteq T_{i}$ such that the $c_{0}$-color of a tuple on any splitting level is determined by restricting to the 0-th splitting level.

\section{Derived TREeS}

This section introduces derived trees and proves a theorem which will be central to the results in Section 4 about small forcings preserving various forms of the Halpern-Läuchli Theorem.

Definition 3.1. Let $\kappa$ be a cardinal, $\mathbb{P}$ be a forcing, and without loss of generality, assume that $\mathbb{P}$ has a largest member, denoted 1 . Assume that $\dot{T}$ is a $\mathbb{P}$-name for which 1 forces that $\dot{T}$ is a subtree of $<\check{\kappa} \check{\kappa}$. The derived tree of $\dot{T}$, denoted $\operatorname{Der}(\dot{T})$, is defined as follows. The elements of $\operatorname{Der}(\dot{T})$ are equivalence classes of pairs $(\dot{\tau}, \alpha)$ satisfying

$$
1 \Vdash(\dot{\tau} \in \dot{T} \text { and } \operatorname{Dom}(\dot{\tau})=\check{\alpha}),
$$

where the equivalence relation $\cong$ is defined by

$$
\left(\dot{\tau}_{1}, \alpha_{1}\right) \cong\left(\dot{\tau}_{2}, \alpha_{2}\right) \Longleftrightarrow 1 \Vdash\left(\dot{\tau}_{1}=\dot{\tau}_{2}\right) .
$$

Notice that if $\left(\dot{\tau}_{1}, \alpha_{1}\right) \cong\left(\dot{\tau}_{2}, \alpha_{2}\right)$, then $\alpha_{1}=\alpha_{2}$. The elements of $\operatorname{Der}(\dot{T})$ are ordered as follows:

$$
\left[\left(\dot{\tau}_{1}, \alpha_{1}\right)\right]<\left[\left(\dot{\tau}_{2}, \alpha_{2}\right)\right] \Longleftrightarrow 1 \Vdash\left(\dot{\tau}_{1} \sqsubseteq \dot{\tau}_{2} \text { and } \dot{\tau}_{1} \neq \dot{\tau}_{2}\right) .
$$

Given $S \subseteq \operatorname{Der}(\dot{T})$, let

$$
\operatorname{Names}(S)=\{\dot{\tau}:(\exists \alpha)[(\dot{\tau}, \alpha)] \in S\} .
$$

We claim that 1 forces that every element of $\dot{T}$ is equal to some element of $\operatorname{Names}(\operatorname{Der}(\dot{T}))$. To see why, let $G$ be $\mathbb{P}$-generic over $V$. Let $t \in \dot{T}_{G}$ and $\alpha=\operatorname{Dom}(t)$. Fix a name $\dot{\tau}$ such that $\dot{\tau}_{G}=t$, and let $\dot{b}$ be a name for the leftmost branch of $\dot{T}$. Define $\dot{\rho}$ so that

$$
1 \Vdash[(\dot{\rho}=\dot{\tau} \text { if } \operatorname{Dom}(\dot{\tau})=\check{\alpha}) \wedge(\dot{\rho}=\dot{b} \uparrow \alpha \text { if } \operatorname{Dom}(\dot{\tau}) \neq \check{\alpha})] .
$$

Then $[(\dot{\rho}, \alpha)] \in \operatorname{Der}(\dot{T})$ and $\dot{\rho}_{G}=t$.

We will now show that $\operatorname{Der}(\dot{T})$ is (isomorphic to) a regular $\kappa$-tree in the ground model whenever 1 forces that $\dot{T}$ is a regular $\kappa$-tree, and that given an element named by some $(\dot{\tau}, \alpha) \in \operatorname{Der}(\dot{T})$, all its successors are 
named by successors of $(\dot{\tau}, \alpha)$ in $\operatorname{Der}(\dot{T})$. This theorem is central to the forcing preservation theorems in following sections.

Given a tree $T \subseteq{ }^{<\kappa} \kappa$ and a node $t \in T$, by the 0 -th successor of $t$ we mean the node $t \frown \alpha \in T$ with the least possible $\alpha \in \kappa$. More generally, the $\gamma$-th successor of $t \in T$ is the node $t^{\frown} \alpha \in T$ such that the set $\left\{\beta<\alpha: t^{\frown} \beta \in T\right\}$ has order type $\gamma$.

Theorem 3.2 (Derived Tree Theorem). Let $\kappa$ be strongly inaccessible, $\mathbb{P}$ a forcing of size $<\kappa$, and $\dot{T}$ a name for a regular $\kappa$-tree. Then $\operatorname{Der}(\dot{T})$ is isomorphic to a regular $\kappa$-tree and

(*) If $[(\dot{\tau}, \alpha)] \in \operatorname{Der}(\dot{T})$ and $X$ is the set of all $\dot{\rho}$ such that $[(\dot{\rho}, \alpha+1)]$ is a successor of $[(\dot{\tau}, \alpha)]$ in $\operatorname{Der}(\dot{T})$, then $1 \Vdash$ (every successor of $\dot{\tau}$ in $\dot{T}$ is named by an element of $\check{X})$.

Proof. First note that if $[(\dot{\tau}, \alpha)]$ is in $\operatorname{Der}(\dot{T})$ and $\beta<\alpha$, then there is a name $\dot{\tau}_{\beta}$ such that $\left[\left(\dot{\tau}_{\beta}, \beta\right)\right]$ is in $\operatorname{Der}(\dot{T})$ and $\left[\left(\dot{\tau}_{\beta}, \beta\right)\right]<[(\dot{\tau}, \alpha)]$ : simply let $\dot{\tau}_{\beta}$ be a name for $\dot{\tau} \uparrow \breve{\beta}$. We prove that $\operatorname{Der}(\dot{T})$ is a regular $\kappa$-tree by proving it satisfies conditions (1) - (3) of Definition 2.1.

To verify (1), we must first show that $\operatorname{Der}(\dot{T})$ is a tree. Suppose $\left[\left(\dot{\tau}_{1}, \alpha_{1}\right)\right],\left[\left(\dot{\tau}_{2}, \alpha_{2}\right)\right],\left[\left(\dot{\tau}_{3}, \alpha_{3}\right)\right]$ are members of $\operatorname{Der}(\dot{T})$ satisfying

$$
\left[\left(\dot{\tau}_{1}, \alpha_{1}\right)\right]>\left[\left(\dot{\tau}_{2}, \alpha_{2}\right)\right] \text { and }\left[\left(\dot{\tau}_{1}, \alpha_{1}\right)\right]>\left[\left(\dot{\tau}_{3}, \alpha_{3}\right)\right] \text {. }
$$

Assume, without loss of generality, that $\alpha_{2} \geq \alpha_{3}$. Since 1 forces that $\dot{T}$ is a tree and that $\dot{\tau}_{i}$ is an initial segment of $\dot{\tau}_{1}$ of length $\alpha_{i}$, for $i \in\{2,3\}$, it follows that 1 forces that $\dot{\tau}_{2}\left\lceil\alpha_{3}=\dot{\tau}_{3}\right.$. Thus, 1 forces that $\dot{\tau}_{3}$ is an initial segment of $\dot{\tau}_{2}$, and hence, $\left[\left(\dot{\tau}_{2}, \alpha_{2}\right)\right]$ and $\left[\left(\dot{\tau}_{3}, \alpha_{2}\right)\right]$ are comparable in $\operatorname{Der}(\dot{T})$.

For $\beta<\kappa$, let level $\beta$ denote the set of those $[(\dot{\tau}, \alpha)] \in \operatorname{Der}(\dot{T})$ such that $\alpha=\beta$. The same argument as above also shows that given $[(\dot{\tau}, \alpha)]$ in $\operatorname{Der}(\dot{T})$ and $\beta<\alpha$, there is a unique $[(\dot{\rho}, \beta)]$ on level $\beta$ of $\operatorname{Der}(\dot{T})$ such that $[(\dot{\rho}, \beta)]<[(\dot{\tau}, \alpha)]$. We have now established that $\operatorname{Der}(\dot{T})$ is a tree.

We must now show that $\operatorname{Der}(\dot{T})$ is a $\kappa$-tree. To show that it has height $\kappa$, given any $\alpha<\kappa$, let $\dot{\tau}_{\alpha}$ be such that $1 \Vdash\left(\dot{\tau}_{\alpha}=\dot{b}\lceil\check{\alpha})\right.$, where $\dot{b}$ is a name for the leftmost branch of $\dot{T}$. Then $\left[\left(\dot{\tau}_{\alpha}, \alpha\right)\right] \in \operatorname{Der}(\dot{T})$. Thus, $\operatorname{Der}(\dot{T})$ has height $\kappa$. To show that each level of $\operatorname{Der}(\dot{T})$ has $<\kappa$ nodes, we will make use of the fact that $\operatorname{Der}(\dot{T})$ consists of elements $[(\dot{\tau}, \alpha)]$ where $1 \Vdash(\operatorname{Dom}(\dot{\tau})=\check{\alpha})$. (If we drop the $\alpha$ 's from the definition of $\operatorname{Der}(\dot{T})$, we can verify $(*)$, and $(2)$ and $(3)$ of Definition 2.1 , but not (1).) Since $1 \Vdash(\dot{T}$ is a $\check{\kappa}$-tree $)$, we have that

$$
1 \Vdash(\forall \alpha<\check{\kappa})(\exists \gamma<\check{\kappa})(\forall t \in \dot{T}) \alpha \in \operatorname{Dom}(t) \Rightarrow t(\alpha)<\gamma .
$$


Since $|\mathbb{P}|<\kappa$, there is a function $g: \kappa \rightarrow \kappa$ such that

$$
1 \Vdash(\forall \alpha<\check{\kappa})(\forall t \in \dot{T}) \alpha \in \operatorname{Dom}(t) \Rightarrow t(\alpha)<\check{g}(\alpha) .
$$

Now, to each pair $(\dot{\tau}, \alpha)$, where $[(\dot{\tau}, \alpha)] \in \operatorname{Der}(\dot{T})$, we may associate a sequence $\left\langle f_{\xi}: \xi<\alpha\right\rangle$, where each $f_{\xi}$ is a function from some maximal antichain of $\mathbb{P}$ to $g(\xi)$. This sequence represents a nice name for $\dot{\tau}$. Since $|\mathbb{P}|<\kappa$, level $\alpha<\kappa$ of $\operatorname{Der}(\dot{T})$ is bounded from above by the following:

$$
\prod_{\xi<\alpha} g(\xi)^{|\mathbb{P}|}
$$

Since $\kappa$ is strongly inaccessible, this bound is $<\kappa$. We have now shown (1) of Definition 2.1.

We will now verify $(2)$, that $\operatorname{Det}(\dot{T})$ has no maximal branches of length $<\kappa$. When we later show that $\operatorname{Der}(\dot{T})$ is perfect, this will imply it has no maximal branches of a successor ordinal length. Thus, it suffices to show $\operatorname{Der}(\dot{T})$ has no maximal branches of limit length. Let $\eta<\kappa$ be a limit ordinal and $S=\left\langle\left[\left(\dot{\tau}_{\alpha}, \alpha\right)\right]: \alpha<\eta\right\rangle$ is an increasing chain in $\operatorname{Der}(\dot{T})$ so that for all $\xi<\zeta<\eta$,

$$
\left[\left(\dot{\tau}_{\xi}, \alpha_{\xi}\right)\right]<\left[\left(\dot{\tau}_{\zeta}, \alpha_{\zeta}\right)\right]
$$

Let $\dot{s}$ be a name which $\mathbb{P}$ forces to be a function from $\check{\eta}$ to $<\check{\kappa} \check{\kappa}$ such that for all $\alpha<\eta$,

$$
1 \Vdash \dot{s}(\check{\alpha})=\dot{\tau}_{\alpha}
$$

By the definition of the tree relation $<$ in $\operatorname{Der}(\dot{T})$, it follows that

$$
1 \Vdash(\forall \alpha<\beta<\check{\eta}) \dot{s}(\alpha) \sqsubseteq \dot{s}(\beta) .
$$

Now let $\dot{\tau}_{\eta}$ be a name such that $1 \Vdash \dot{\tau}_{\eta}=\bigcup_{\alpha<\check{\eta}} \dot{s}(\alpha)$. It follows from (15) that

$$
1 \Vdash \dot{\tau}_{\eta} \in \check{\eta}_{\check{\kappa}} \text { and }(\forall \alpha<\check{\eta}) \dot{s}(\alpha) \sqsubseteq \dot{\tau}_{\eta},
$$

and thus, by (14),

$$
1 \Vdash(\forall \alpha<\check{\eta}) \dot{\tau}_{\alpha} \sqsubseteq \dot{\tau}_{\eta}
$$

Since $\mathbb{P}$ forces that $\dot{T}$ has no maximal branches of length $<\kappa$, we now have that

$$
1 \Vdash \dot{\tau}_{\eta} \in \dot{T}
$$

So now $\left[\left(\dot{\tau}_{\eta}, \eta\right)\right] \in \operatorname{Der}(\dot{T})$, and this node is above each $\left[\left(\dot{\tau}_{\alpha}, \alpha\right)\right]$ for $\alpha<\eta$. Thus, we have verified (2) of Definition 2.1.

To verify $(3)$, consider any $[(\dot{\tau}, \alpha)] \in \operatorname{Der}(\dot{T})$. Let $\dot{b}$ be a name for the leftmost branch of $\dot{T}$ which extends $\dot{\tau}$. Let $\beta<\kappa$ be the least 
ordinal greater than or equal to $\alpha$ for which there is some $p \in \mathbb{P}$ which forces that there are at least two successors of $\dot{b} \uparrow \check{\beta}$ in the tree $\dot{T}$. Such $\beta$ and $p$ exist since $\mathbb{P}$ forces that $\dot{T}$ is a perfect tree. Let $\dot{\tau}_{1}$ be a name such that 1 forces $\dot{\tau}_{1}=\dot{b} \uparrow \check{\beta}$. Let $\dot{\tau}_{2}$ be a name which 1 forces to be the 0 -th successor of $\dot{\tau}_{1}$ in $\dot{T}$. Finally, let $\dot{\tau}_{3}$ be a name which 1 forces to be the 1-th successor of $\dot{\tau}_{1}$ in $\dot{T}$, if there are at least two successors, and the unique successor if there is only one successor. One can see that $\left[\left(\dot{\tau}_{2}, \beta+1\right)\right]$ and $\left[\left(\dot{\tau}_{3}, \beta+1\right)\right]$ are successors of $\left[\left(\dot{\tau}_{1}, \beta\right)\right]$ in $\operatorname{Der}(\dot{T})$. Since there is some $p$ which forces $\dot{\tau}_{2} \neq \dot{\tau}_{3}$, it follows that $\left[\left(\dot{\tau}_{2}, \beta+1\right)\right] \neq\left[\left(\dot{\tau}_{3}, \beta+1\right)\right]$. Thus, $\left[\left(\dot{\tau}_{2}, \beta+1\right)\right]$ and $\left[\left(\dot{\tau}_{3}, \beta+1\right)\right]$ are incomparable extensions of $[(\dot{\tau}, \alpha)]$ in $\operatorname{Der}(\dot{T})$. Therefore, $\operatorname{Der}(\dot{T})$ is a perfect tree. Hence, $\operatorname{Der}(\dot{T})$ is isomorphic to a regular $\kappa$-tree.

Finally, the verification of $(*)$ follows almost immediately from the definition of $\operatorname{Der}(\dot{T})$. Fix $[(\dot{\tau}, \alpha)] \in \operatorname{Der}(\dot{T})$ and let $G$ be $\mathbb{P}$-generic over $V$. Let $s$ be an arbitrary successor of $\dot{\tau}_{G}$ in $\dot{T}_{G}$, and let $\gamma$ be such that $s$ is the $\gamma$-th successor of $\dot{\tau}_{G}$ in $\dot{T}_{G}$. Take $\dot{\rho}$ to be a name so that 1 forces that $\dot{\rho}$ is the $\check{\gamma}$-th successor of $\dot{\tau}$ in $\dot{T}$, if it exists, and the 0 -th successor, otherwise. Then $\dot{\rho}_{G}=s$. At the same time 1 forces that $\dot{\rho}$ is a successor of $\dot{\tau}$ in $\dot{T}$, so $[(\dot{\rho}, \alpha+1)]$ is a member of $\operatorname{Der}(\dot{T})$.

Remark 3.3. There are two instances in the proof where $|\mathbb{P}|<\kappa$ was used. The first is non-essential: If $\mathbb{P}$ is $\kappa$-c.c., or even just $(\kappa, \kappa,<$ $\kappa)$-distributive, equation (11) still holds. However, the second use of $|\mathbb{P}|<\kappa$ is essential to the proof. Indeed, given any $\mathbb{P}$ which preserves $\kappa$ and has cardinality at least $\kappa$, there is a name $\dot{T}$ for a regular $\kappa$-tree with the following properties: 1 forces that the first level of $\dot{T}$ has size at least two (with say elements $\langle 0\rangle$ and $\langle 1\rangle$ ), and letting $\left\{p_{\zeta}: \zeta<\kappa\right\}$ be a set of distinct members of $\mathbb{P}$, there are nice names $\dot{\tau}_{\zeta}$ so that

$$
p_{\zeta} \Vdash \dot{\tau}_{\zeta} \in \dot{T}, \operatorname{Dom}\left(\dot{\tau}_{\zeta}\right)=1 \text {, and } \dot{\tau}_{\zeta}(0)=0 \text {, }
$$

and all $q \in \mathbb{P}$ incompatible with $p_{\zeta}$ force $\dot{\tau}_{\zeta}(0)=1$. Then for all $\zeta<\xi<\kappa,\left(\dot{\tau}_{\zeta}, 1\right) \nRightarrow\left(\dot{\tau}_{\xi}, 1\right)$, so the first level of $\operatorname{Der}(\dot{T})$ has size at least $\kappa$. Thus, the $\kappa$-c.c. is not enough to guarantee that the levels of $\operatorname{Der}(\dot{T})$ have size less than $\kappa$.

The Derived Tree Theorem will allow us to use $\operatorname{Der}(\dot{T})$ in theorems that require subtrees of ${ }^{<\kappa} \kappa$. Note also that the Derived Tree Theorem provides the following: Given a name for a regular $\kappa$-tree $\dot{T}$, a strong subtree $S$ of $\operatorname{Der}(\dot{T})$ with splitting levels $A \subseteq \kappa$, and a $\mathbb{P}$-generic $G$, the set $W=\left\{\dot{\tau}_{G}: \dot{\tau} \in \operatorname{Names}(S)\right\}$ is a strong subtree of $\dot{T}_{G}$, witnessed by the set of splitting levels $A$. 
4. Small Forcings Preserve SDHL, HL, And HL ${ }^{t c}$

In this section we show that if $\kappa$ is strongly inaccessible and $(\forall \sigma<$ $\kappa) \operatorname{SDHL}(d, \sigma, \kappa)$ holds, then this still holds after performing any forcing of size less than $\kappa$. This result then automatically holds for HL replacing SDHL, since the two are equivalent for $\kappa$ inaccessible. Further, we show that $\mathrm{HL}^{t c}$ at $\kappa$ is preserved by forcings of size less than $\kappa$. These results make strong use of the Derived Tree Theorem from the previous section.

Theorem 4.1. Let $\kappa$ be strongly inaccessible. Let $1 \leq d<\omega$ and $0<$ $\sigma<\kappa$. Let $\mathbb{P}$ be a forcing of size $<\kappa$. Assume that $\operatorname{SDHL}(d, \sigma \cdot|\mathbb{P}|, \kappa)$ holds. Then $S D H L(d, \sigma, \kappa)$ holds after forcing with $\mathbb{P}$. In particular, the statement " $\forall \sigma<\kappa) S D H L(d, \sigma, \kappa)$ holds" is preserved by all forcings of size less than $\kappa$.

Proof. Let $\left\langle\dot{T}_{i}: i<d\right\rangle$ be a sequence of names for regular trees in the extension. That is, $(\forall i<d) 1 \Vdash\left(\dot{T}_{i} \subseteq<\check{\kappa}_{\breve{\kappa}}\right.$ is regular $)$. Let $\dot{c}$ be such that

$$
1 \Vdash \dot{c}: \bigotimes_{i<\check{d}} \dot{T}_{i} \rightarrow \check{\sigma}
$$

We must show that $\mathbb{P}$ forces that there is a somewhere dense level matrix $\left\langle X_{i} \subseteq \dot{T}_{i}: i<\check{d}\right\rangle$ such that $\left|\dot{c}^{"} \bigotimes_{i<\check{d}} X_{i}\right|=1$. We will do this by showing that for each $p \in \mathbb{P}$, there is some $p^{\prime} \leq p$ forcing this statement. Fix $p \in \mathbb{P}$.

Consider the trees $\operatorname{Der}\left(\dot{T}_{i}\right)$ for $i<d$. Let

$$
c^{\prime}: \bigotimes_{i<d} \operatorname{Der}\left(\dot{T}_{i}\right) \rightarrow \sigma \times \mathbb{P}
$$

be a coloring defined so that for any $\alpha<\kappa$ and any level $d$-tuple

$$
\vec{t}=\left\langle\left[\left(\dot{\tau}_{i}, \alpha\right)\right] \in \operatorname{Der}\left(\dot{T}_{i}\right): i<d\right\rangle,
$$

$c^{\prime}(\vec{t})=\left\langle\sigma^{\prime}, q\right\rangle$ where $\sigma^{\prime}$ and $q$ satisfy $q \leq p$ and

$$
q \Vdash \dot{c}\left\langle\dot{\tau}_{i}: i<d\right\rangle=\check{\sigma}^{\prime} .
$$

That is, the $c^{\prime}$-color of $\vec{t}$ is a pair $\left\langle\sigma^{\prime}, q\right\rangle \in \sigma \times \mathbb{P}$ where $q$ forces the $\dot{c}$-color of the tuple named by $\vec{t}$ to have the color $\check{\sigma}^{\prime}$.

Since $\operatorname{SDHL}(d, \sigma \cdot|\mathbb{P}|, \kappa)$ holds, there is a somewhere dense level ma$\operatorname{trix}\left\langle Y_{i} \subseteq \operatorname{Der}\left(\dot{T}_{i}\right): i<d\right\rangle$ that is $c^{\prime}$-monochromatic. Let $\xi<\kappa$ be such that each $Y_{i}$ is on level $\xi$ of $\operatorname{Der}(\dot{T})$. Fix $\zeta<\xi$ and for each $i<d$, fix $\left[\left(\dot{\delta}_{i}, \zeta\right)\right] \in \operatorname{Der}(\dot{T})$ such that $Y_{i}$ dominates the set of successors of $\left[\left(\dot{\delta}_{i}, \zeta\right)\right]$ 
in $\operatorname{Der}\left(\dot{T}_{i}\right)$. Let $\left\langle\sigma^{\prime}, p^{\prime}\right\rangle$ be the unique color assigned to each element of $\bigotimes_{i<d} Y_{i}$ by $c^{\prime}$. Hence for all $\left\langle\left[\left(\dot{\tau}_{i}, \xi\right)\right] \in Y_{i}: i<d\right\rangle$,

$$
p^{\prime} \Vdash \dot{c}\left\langle\dot{\tau}_{i}: i<d\right\rangle=\check{\sigma}^{\prime}
$$

We now show that $p^{\prime}$ forces that there is a $\dot{c}$-monochromatic somewhere dense level matrix of $\left\langle\dot{T}_{i}: i<d\right\rangle$. Let $G$ be any $\mathbb{P}$-generic over $V$ containing $p^{\prime}$. It suffices to show that in $V[G]$, there is a $\dot{c}_{G^{-}}$ monochromatic somewhere dense level matrix of $\left\langle\left(\dot{T}_{i}\right)_{G}: i<d\right\rangle$.

For each $i<d$, let

$$
X_{i}=\left\{\dot{\tau}_{G}: \dot{\tau} \in \operatorname{Names}\left(Y_{i}\right)\right\} .
$$

By $(*)$ of Theorem 3.2 ,

$$
(\forall i<d) X_{i} \text { dominates the successors of }\left(\dot{\delta}_{i}\right)_{G} \text { in }\left(\dot{T}_{i}\right)_{G},
$$

so $\left\langle X_{i}: i<d\right\rangle$ is a somewhere dense level matrix of $\left\langle\left(T_{i}\right)_{G}: i<d\right\rangle$. By (24) and since $p^{\prime} \in G$, we have that

$$
\dot{c}_{G} " \bigotimes_{i<d} X_{i}=\left\{\sigma^{\prime}\right\}
$$

so $\left\langle X_{i}: i<d\right\rangle$ is $\dot{c}_{G}$-monochromatic.

The Derived Tree Theorem also implies that $\mathrm{HL}^{t c}$ is preserved by small forcings, as we will now see.

Theorem 4.2. Let $\kappa$ be strongly inaccessible. Let $1 \leq d<\omega$. Let $\mathbb{P}$ be a forcing of size $<\kappa$. Assume that $H L^{t c}(d,<\kappa, \kappa)$ holds. Then $H L^{t c}(d,<\kappa, \kappa)$ holds after forcing with $\mathbb{P}$.

Proof. The proof is similar to that of the previous theorem. Fix $p \in \mathbb{P}$. By Proposition 2.11, it suffices to find a $p^{\prime} \leq p$ that forces the modified version of $\mathrm{HL}^{t c}$. Let $\left\langle\dot{T}_{i}: i<d\right\rangle$ be a sequence of names for regular trees, and let $\left\langle\dot{c}_{\zeta}: \zeta<\kappa\right\rangle$ be a sequence of names for colorings such that $\mathbb{P}$ forces each $\dot{c}_{\zeta}$ to take less than $\check{\kappa}$ colors. Since $|\mathbb{P}|<\kappa$, there are ordinals $\sigma_{\zeta}<\kappa$ for $\zeta<\kappa$ such that

$$
1 \Vdash \dot{c}_{\zeta}: \bigotimes_{i<\check{d}} \dot{T}_{i} \rightarrow \check{\sigma}_{\zeta}
$$

Just as in the previous theorem, the sequence of colorings $\left\langle\dot{c}_{\zeta}: \zeta<\kappa\right\rangle$ induces a sequence of colorings $\left\langle c_{\zeta}^{\prime}: \zeta<\kappa\right\rangle$ where for each $\zeta<\kappa$,

$$
c_{\zeta}^{\prime}: \bigotimes_{i<d} \operatorname{Der}\left(\dot{T}_{i}\right) \rightarrow \sigma_{\zeta} \times \mathbb{P}
$$

Now apply $\operatorname{HL}^{t c}(d,<\kappa, \kappa)$ to the sequence of the trees $\left\langle\operatorname{Der}\left(\dot{T}_{i}\right): i<d\right\rangle$ and the sequence of colorings $\left\langle c_{\zeta}^{\prime}: \zeta<\kappa\right\rangle$. What results is a sequence 
of strong subtrees $S_{i} \subseteq \operatorname{Der}\left(\dot{T}_{i}\right)$ for $i<d$, all witnessed by the same set of splitting levels $A \subseteq \kappa$. Let $A$ be enumerated in increasing order as $A=\left\{\alpha_{\zeta}: \zeta<\kappa\right\}$. For each pair of ordinals $\zeta \leq \xi<\kappa$, given any $d$-tuple $\vec{t} \in \bigotimes_{i<d} S_{i}\left(a_{\xi}\right)$, the $c_{\zeta}^{\prime}$-th color of that tuple is the same as the $c_{\zeta}^{\prime}$-th color of that tuple restricted to $\bigotimes_{i<d} S_{i}\left(\alpha_{\zeta}\right)$.

Recall that for the colorings $\left\langle c_{\zeta}^{\prime}: \zeta<\kappa\right\rangle$, the $c_{\zeta}^{\prime}$-th color of a tuple is a pair $\left\langle\sigma^{\prime}, q\right\rangle$. If the $q$-component of all the tuples from the splitting levels of the $S_{i}$ trees are the same, then that $q$ forces that the colorings are homogenized in the desired way. In that case, we can set $p^{\prime}=q$ and be done. So, the challenge now is to further homogenize to make the $q$ 's the same.

Let $P: \bigcup_{\zeta<\kappa} \bigotimes_{i<d} S_{i}\left(\alpha_{\zeta}\right) \rightarrow \mathbb{P}$ be the following coloring: Given $\zeta<\kappa$ and $\vec{t}=\left\langle t_{i} \in S_{i}\left(\alpha_{\zeta}\right): i<d\right\rangle$, define

$$
P(\vec{t})=\text { the } q \text {-component of } c_{\zeta}^{\prime}\left\langle t_{i}: i<d\right\rangle \text {. }
$$

We mentioned in Observation 2.12 that $\operatorname{HL}^{t c}(d,<\kappa, \kappa)$ implies $\operatorname{HL}(d, \sigma, \kappa)$ for all $\sigma<\kappa$. Apply $\operatorname{HL}(d,|\mathbb{P}|, \kappa)$ to obtain trees $U_{i} \subseteq S_{i}$ for $i<d$, such that each $U_{i}$ is a strong subtree of $T_{i}$ as witnessed by a set of splitting levels $B \subseteq A$, such that for some fixed $p^{\prime}$,

$$
P^{*} \bigcup_{\beta \in B} \bigotimes_{i<d} U_{i}(\beta)=\left\{p^{\prime}\right\} \text {. }
$$

Now we have that for any two ordinals $\zeta \leq \xi<\kappa$ such that $\alpha_{\zeta} \in B$, given any $\vec{t}=\left\langle\left[\left(\dot{\tau}_{i}, \alpha_{\xi}\right)\right] \in U_{i}\left(\alpha_{\xi}\right): i<d\right\rangle$,

$$
p^{\prime} \Vdash \dot{c}_{\zeta}\left\langle\dot{\tau}_{i}: i<d\right\rangle=\dot{c}_{\zeta}\left\langle\dot{\tau}_{i} \uparrow \alpha_{\zeta}: i<d\right\rangle .
$$

We are now almost done. The only problem is that each $\alpha_{\zeta}$ is not necessarily the $\zeta$-th splitting level of the $U_{i}$ 's (recall that $\alpha_{\zeta}$ is the $\zeta$-th splitting level of the $S_{i}$ 's). Now let $\left\{\beta_{\gamma}: \gamma<\kappa\right\}=B$ be the increasing enumeration of $B$. Let $\mu: \kappa \rightarrow \kappa$ be the function such that

$$
\beta_{\gamma}=\alpha_{\mu(\gamma)}
$$

The function $\mu$ is strictly increasing and $\gamma \leq \mu(\gamma)$ for all $\gamma<\kappa$. Using (32) and substituting into equation (31), we obtain that for any given $\gamma \leq \nu<\kappa$ and $\vec{t}=\left\langle\left[\left(\dot{\tau}_{i}, \beta_{\nu}\right)\right] \in U_{i}\left(\beta_{\nu}\right): i<d\right\rangle$,

$$
p^{\prime} \Vdash \dot{c}_{\mu(\gamma)}\left\langle\dot{\tau}_{i}: i<d\right\rangle=\dot{c}_{\mu(\gamma)}\left\langle\dot{\tau}_{i} \uparrow \beta_{\gamma}: i<d\right\rangle .
$$

That is, $p^{\prime}$ forces that the $\dot{c}_{\mu(\gamma)}$-color is determined by restricting to level $\beta_{\gamma}$, the $\gamma$-th splitting level of the $U_{i}$ 's.

Now let $G$ be $\mathbb{P}$-generic over $V$ such that $q^{\prime} \in G$. For each $i<d$, let

$$
W_{i}=\left\{\dot{\tau}_{G}: \dot{\tau} \in \operatorname{Names}\left(U_{i}\right)\right\}
$$


By our comments following Theorem 3.2 , each $W_{i}$ is a strong subtree of $\left(T_{i}\right)_{G}$. Since $q^{\prime}$ is in $G$ and (33) holds, the $\left(\dot{c}_{\mu(\gamma)}\right)_{G}$-color of a tuple from $\bigcup_{\beta \in B} \bigoplus_{i<d} W_{i}(\beta)$ is determined by restricting to the $\gamma$-th splitting level $\beta_{\gamma}$ of the $W_{i}$ 's. Thus, the conclusion of the modified $\mathrm{HL}^{t c}$ holds in $V[G]$. This completes the proof.

Remark 4.3. Let $\kappa$ be strongly inaccessible. There is a unique $\kappa$ saturated linear order of size $\kappa$ denoted $\mathbb{Q}_{\kappa}$, the $\kappa$-rationals [17]. Zhang proved in [17] that $\mathrm{HL}^{t c}(d,<\kappa, \kappa)$ implies

$$
\left(\begin{array}{c}
\mathbb{Q}_{\kappa} \\
\vdots \\
\mathbb{Q}_{\kappa}
\end{array}\right) \rightarrow\left(\begin{array}{c}
\mathbb{Q}_{\kappa} \\
\vdots \\
\mathbb{Q}_{\kappa}
\end{array}\right) \underbrace{\underbrace{1, \ldots, 1}_{d+1}}_{<\kappa,(d+1) !}
$$

This partition relation means that given any $\sigma<\kappa$ and any coloring $c: \prod_{i<d+1} \mathbb{Q}_{\kappa} \rightarrow \sigma$, there is a sequence $\left\langle X_{i} \subseteq \mathbb{Q}_{\kappa}: i<d+1\right\rangle$, where each $X_{i}$ is order isomorphic to $\mathbb{Q}_{\kappa}$, such that

$$
\left|c^{*} \prod_{i<d+1} X_{i}\right| \leq(d+1) !
$$

Thus, whenever $V$ satisfies $\mathrm{HL}^{t c}(d,<\kappa, \kappa)$, combining Theorem 4.2 with Zhang's result yields that every forcing extension of $V$ by a poset of size less than $\kappa$ satisfies the partition relation (34).

In contrast, other partition relations are falsified by small forcings. For example in [6], Hajnal and Komjáth define a fixed poset $\mathbb{K}$ that forces the following for sufficiently large $\kappa$ :

$$
\mathbb{Q}_{\kappa} \nrightarrow \rightarrow\left[\mathbb{Q}_{\kappa}\right]_{\omega}^{2} \text {. }
$$

This partition relation means that there exsits a coloring $c:\left[\mathbb{Q}_{\kappa}\right]^{2} \rightarrow \omega$ such that for any set $X \subseteq \mathbb{Q}_{\kappa}$ of order type $\mathbb{Q}_{\kappa}$, we have

$$
f^{"}[X]^{2}=\omega .
$$

That is, there is a coloring of the pairs from $\mathbb{Q}_{\kappa}$ using $\omega$ colors such that no set order isomorphic to $\mathbb{Q}_{\kappa}$ omits any color.

More specifically, Hajnal and Komjáth show that that assuming there are no Suslin trees of height $\omega_{1}$ (which can be forced by a small forcing), then after adding a Cohen real, there is a linear ordering $\theta$ of size $\omega_{1}$ such that for any linear ordering $\Omega$, there is a coloring $c:[\Omega]^{2} \rightarrow \omega$ such that every subset of $\Omega$ order isomorphic to $\theta$ does not omit any color. 


\section{REFLECTION}

At inaccessible cardinals, the Halpern-Läuchli Theorem reflects. In Proposition 5.1, we show that for $\kappa$ strongly inaccessible, if SDHL holds on a stationary set below $\kappa$, then it holds at $\kappa$. In this proposition, SDHL cannot be replaced by $\mathrm{HL}^{t c}$, which we will explain in the next paragraph. In Proposition 5.2, we prove that SDHL holds at a measurable cardinal $\kappa$ if and only if the set of ordinals below $\kappa$ where SDHL holds is a member of any normal ultrafilter on $\kappa$. By Proposition 2.8, the same statement holds for HL. It also holds for $\mathrm{HL}^{t c}$. These two propositions imply Theorem 5.3, that the Halpern-Läuchli Theorem at a measurable cardinal $\kappa$ is preserved by $<\kappa$-closed forcings.

Let us explain why Proposition 5.1 does not hold for $\mathrm{HL}^{t c}$. The problem is we could use the argument of Theorem 6.3 in the next section to get $\mathrm{HL}^{t c}$ to hold at a cardinal that is not weakly compact, which is impossible by [17]. That is, assume Proposition 5.1 does hold for $\mathrm{HL}^{t c}$ and start with $V$ satisfying $\mathrm{HL}^{t c}$ at a measurable $\kappa$. Then perform any nontrivial forcing of size less than $\kappa$ to obtain some generic extension $V[G]$. In $V[G], \kappa$ is still measurable and $\mathrm{HL}^{t c}$ holds at $\kappa$. So in $V[G], \mathrm{HL}^{t c}$ holds for a stationary set of $\lambda<\kappa$. Now let $V[G][H]$ be any nontrivial forcing extension of $V[G]$ by a $<\kappa$-closed forcing. Then in $V[G][H], \mathrm{HL}^{t c}$ holds for a stationary set of $\lambda<\kappa$. Since we are assuming Proposition 5.1 holds for $\mathrm{HL}^{t c}$, then in $V[G][H]$, $\mathrm{HL}^{t c}$ holds at $\kappa$. This is impossible, because by a result of Hamkins [9] any nontrivial forcing of size less than $\kappa$ followed by any nontrivial $<\kappa$-closed forcing causes $\kappa$ to not be weakly compact.

Proposition 5.1. Let $\kappa$ be a cardinal such that either

- $\kappa$ is strongly inaccessible or

- $c f(\kappa) \geq \omega_{1}$ and $\kappa$ is the limit of strongly inaccessible cardinals.

Let $1 \leq d<\omega$ and $1 \leq \sigma<\kappa$, and assume that $\operatorname{SDHL}(d, \sigma, \alpha)$ holds for a stationary subset of $\kappa$. Then $\operatorname{SDHL}(d, \sigma, \kappa)$ holds.

Proof. Let $\left\langle T_{i}: i<d\right\rangle$ be a sequence of regular $\kappa$-trees and let $c$ : $\bigotimes_{i<d} T_{i} \rightarrow \sigma$ be a coloring. If we can find an $\alpha<\kappa$ such that each $T_{i} \cap^{<\alpha} \kappa$ is a regular $\alpha$-tree and $\operatorname{SDHL}(d, \sigma, \alpha)$ holds, then we will be done. This is because a monochromatic somewhere dense level matrix in $\bigotimes_{i<d}\left(T_{i} \cap{ }^{<\alpha} \kappa\right)$, is automatically a monochromatic somewhere dense level matrix in $\bigotimes_{i<d} T_{i}$.

Fix $i<d$. The following standard argument shows that there is a club $C_{i} \subseteq \kappa$ such that for each $\alpha \in C_{i}$, the following hold:

(1) $\alpha$ is a cardinal;

(2) Each level of $T_{i} \cap^{<\alpha} \kappa$ has size less than $\alpha$; 
(3) $T_{i} \cap^{<\alpha} \kappa$ is perfect.

Let $\alpha_{0}=0$ be the least member of $C_{i}$. Given $\alpha_{\gamma}$, the $\gamma$-th member of $C_{i}$, construct $\alpha_{\gamma+1}$ as follows. Let $\lambda_{0}=\alpha_{\gamma}$. Given $\lambda_{n}$ for $n<\omega$, let $\lambda_{n+1}$ be the least cardinal above $\lambda_{n}$ such that both (i) and (ii) hold: (i) For each $\beta<\lambda_{n}, T_{i} \cap{ }^{\beta} \kappa$ is contained in ${ }^{\beta} \lambda_{n+1}$ and has cardinality less than $\lambda_{n+1}$. (ii) For each $t \in T_{i} \cap{ }^{\lambda_{n}} \kappa$, there are at least two incomparable extensions of $t$ in $T_{i} \cap^{<\lambda_{n+1}} \kappa$. Note that $\lambda_{n+1}<\kappa$ by the cardinal assumption on $\kappa$. Now define $\alpha_{\gamma+1}=\sup _{n<\omega} \lambda_{n}$. Since $\operatorname{cf}(\kappa) \geq \omega_{1}$ we have $\alpha_{\gamma+1}<\kappa$. By the construction, $\alpha_{\gamma+1}$ satisfies (1) - (3).

Given a limit ordinal $\gamma<\kappa$ and the increasing sequence $\left\langle\alpha_{\zeta}: \zeta<\gamma\right\rangle$, define $\alpha_{\gamma}=\sup _{\zeta<\gamma} \alpha_{\zeta}$. Note that $\alpha_{\gamma}$ automatically satisfies (1) - (3). Thus, given any $\alpha \in C_{i}, T_{i} \cap{ }^{<\alpha} \kappa$ is a regular $\alpha$-tree. This defines $C_{i}$ as desired, and it is clear that $C_{i}$ is club.

Let $S$ be a stationary subset of $\kappa$ such that $\operatorname{SDHL}(d, \sigma, \alpha)$ holds for each $\alpha \in S$. The set $\bigcap_{i<d} C_{i}$ is a club subset of $\kappa$, so it must intersect $S$ (here we are using that $\operatorname{cf}(\kappa) \geq \omega_{1}$ ). Take any $\alpha<\kappa$ in the intersection. Then $\alpha$ is a cardinal, $T_{i} \cap^{<\alpha} \kappa$ is a regular $\alpha$-tree for each $i<d$, and $\operatorname{SDHL}(d, \sigma, \kappa)$ holds, which is what we intended to show.

For measurable cardinals, we have an even stronger form of reflection using a normal ultrafilter. The same argument works for HL and $\mathrm{HL}^{t c}$, because these are all statements about $V_{\kappa+1}$.

Proposition 5.2. Let $\kappa$ be a measurable cardinal and $\mathcal{U}$ be a normal ultrafilter on $\kappa$. Then

$$
\operatorname{SDHL}(d, \sigma, \kappa) \Leftrightarrow\{\alpha<\kappa: \operatorname{SDHL}(d, \sigma, \alpha)\} \in \mathcal{U} .
$$

Proof. Let $j: V \rightarrow M$ be the ultrapower embedding coming from $\mathcal{U}$. Since $V_{\kappa+1} \subseteq M$,

$$
\operatorname{SDHL}(d, \sigma, \kappa) \Leftrightarrow \operatorname{SDHL}(d, \sigma, \kappa)^{M} .
$$

By Łos's Theorem,

$$
\operatorname{SDHL}(d, \sigma, \kappa)^{M} \Leftrightarrow\{\alpha<\kappa: \operatorname{SDHL}(d, \sigma, \alpha)\} \in \mathcal{U} .
$$

Theorem 5.3. Suppose $\kappa$ is a measurable cardinal. Let $1 \leq d<\omega$ and $1 \leq \sigma<\kappa$ be given, and assume $\operatorname{SDHL}(d, \sigma, \kappa)$ holds. If $\mathbb{P}$ preserves stationary subsets of $\kappa$ and adds no new bounded subsets of $\kappa$, then $\operatorname{SDHL}(d, \sigma, \kappa)$ holds after forcing with $\mathbb{P}$. In particular, if $\mathbb{P}$ is $<\kappa$ closed, then $S D H L(d, \sigma, \kappa)$ holds after forcing with $\mathbb{P}$.

Proof. As $<\kappa$-closed forcings preserve stationary subsets of $\kappa$ and add no new bounded subsets of $\kappa$, we need only prove the first half of the theorem. 
Fix a normal ultrafilter $\mathcal{U}$ on $\kappa$. Since $\operatorname{SDHL}(d, \sigma, \kappa)$ holds, by Proposition 5.2 the set

$$
S=\{\alpha<\kappa: \operatorname{SDHL}(d, \sigma, \alpha)\}
$$

is in $\mathcal{U}$. Hence, $S$ is stationary. Since $\mathbb{P}$ preserves stationary subsets of $\kappa, 1 \Vdash \check{S}$ is stationary. For $\alpha<\kappa$, since $\operatorname{SDHL}(d, \sigma, \alpha)$ is a statement about $V_{\alpha+1}$, and $V_{\alpha+1}$ is the same when computed in the forcing extension by $\mathbb{P}$, we have that $\mathbb{P}$ does not change the truth value of $\operatorname{SDHL}(d, \sigma, \alpha)$ for any $\alpha<\kappa$. So,

$$
1 \Vdash\{\alpha<\check{\kappa}: \operatorname{SDHL}(\check{d}, \check{\sigma}, \alpha)\} \text { is stationary. }
$$

By Proposition 5.1, $\mathbb{P}$ forces that $\operatorname{SDHL}(\check{d}, \check{\sigma}, \check{\kappa})$ holds.

\section{SDHL at a Cardinal That is Not Weakly Compact}

In 2], we proved that $\operatorname{SDHL}(1, k, \kappa)$ holds for all finite $k$ and all infinite cardinals $\kappa$. So, by the equivalence of SDHL and HL for all strongly inaccessible cardinals $\kappa$, $\operatorname{HL}(1, k, \kappa)$ holds for every strongly inaccessible $\kappa$ and every finite $k$. In [17], Zhang showed that this can be improved to $H L^{\text {asym }}(1, \sigma, \kappa)$ holding for all $\sigma<\kappa$, but he required $\kappa$ to be weakly compact. So, it is natural to wonder whether $\kappa>\omega$ needs to be weakly compact in order for $\operatorname{HL}(2, \sigma, \kappa)$ to hold for all $\sigma<\kappa$.

While we were writing [2] we discovered the derived tree theorem and the proof in this section, which answers the question in the negative. In the meantime, Zhang discovered a different proof of the consistency of $(\forall \sigma<\kappa) \operatorname{HL}(2, \sigma, \kappa)$ for a $\kappa$ that is not weakly compact. Specifically, in Theorem 5.8 of [17] he proved that if for all $d<\omega, \kappa$ is measurable whenever one adds $\kappa^{+d}$ many Cohen subsets of $\kappa$, then there is a forcing extension in which $\kappa$ is inaccessible but not weakly compact, and in which $\operatorname{HL}(d, \sigma, \kappa)$ holds for all $d<\omega$ and all $\sigma<\kappa$. The theorem we will present now implies this, but has a different proof and applies to a broad collection of forcings.

Definition 6.1. For $1 \leq d<\omega$ and an infinite cardinal $\kappa, \Psi_{d, \kappa}$ is the statement

$$
(\forall \sigma<\kappa) \operatorname{HL}(d, \sigma, \kappa)
$$

In [2] we showed the following:

Theorem 6.2. Let $1 \leq d<\omega$. If $\kappa$ is measurable whenever one adds $\kappa^{+d}$ many Cohen subsets of $\kappa$, then $\Psi_{d, \kappa}$ holds (in V).

Theorem 6.3. Let $1 \leq d<\omega$ and $\kappa$ be measurable. Assume $\Psi_{d, \kappa}$ holds. Then any non-trivial forcing of size less than $\kappa$ followed by a 
non-trivial $<\kappa$-closed forcing produces a model in which $\kappa$ is not weakly compact and $\Psi_{d, \kappa}$ holds.

Proof. By a theorem of Hamkins [9], any non-trivial forcing of size less than $\kappa$ followed by a non-trivial $<\kappa$-closed forcing will force $\kappa$ to not be weakly compact.

Let $\mathbb{P}$ be any non-trivial forcing of size $<\kappa$. Let $G$ be $\mathbb{P}$-generic over $V$. Then $\Psi_{d, \kappa}$ holds in $V[G]$ by Theorem 4.1. Let $\mathbb{Q}$ be any nontrivial $<\kappa$-closed forcing in $V[G]$, and let $H$ be $\mathbb{Q}$-generic over $V[G]$. By Hamkins's result, $\kappa$ is not weakly compact in $V[G][H]$. Since $\Psi_{d, \kappa}$ holds in $V[G]$ and $\kappa$ is measurable in this model, it follows from Theorem 5.3 that $\Psi_{d, \kappa}$ also holds in $V[G][H]$.

\section{Open Problems}

The main open problem concerning the Halpern-Läuchli Theorem at uncountable cardinals is the following:

Question 7.1. Is it consistent for $\operatorname{HL}(d, \sigma, \kappa)$ to fail for some uncountable cardinal $\kappa$ ?

Because this is unanswered, there are many secondary questions. For example, even though $\operatorname{HL}(d, \sigma, \kappa)$ does not imply $\kappa$ itself must be weakly compact, does it have any large cardinal strength? Does HL have so much large cardinal strength that it cannot hold in L; or does HL always hold in L? Does the existence of say $0^{\#}$ imply that within $L$, HL holds at some, or all, strongly inaccessible cardinals?

In all known models in which $\operatorname{HL}(d, \sigma, \kappa)$ holds for some strongly inaccessible $\kappa$, GCH fails. Such models appear in [14, [4], 5], [2, [17] and the preceding sections of this article. Is $\operatorname{HL}(d, \sigma, \kappa)$ for $\kappa$ strongly inaccessible consistent with GCH?

In this article, we showed that various forms of HL are preserved by small forcings or by $<\kappa$-closed forcings. What other types of forcings preserve HL? An obvious question is the following:

Question 7.2. Do $\kappa$-c.c. forcings preserve $\operatorname{HL}(d, \sigma, \kappa)$, for $0<d<\omega$ and $0<\sigma<\kappa$ ?

Many variants of these questions can be formulated, and progress on any of them will lead to a better understanding of Halpern-Läuchli Theorems and associated partition relations on uncountable structures.

\section{REFERENCES}

1. Dennis Devlin, Some partition theorems for ultrafilters on $\omega$, Ph.D. thesis, Dartmouth College, 1979. 
2. Natasha Dobrinen and Daniel Hathaway, The Halpern-Läuchli Theorem at a measurable cardinal, Journal of Symbolic Logic 82 (2017), no. 4, 1560-1575.

3. Pandelis Dodos and Vassilis Kanellopoulos, Ramsey Theory for Product Spaces, American Mathematical Society, 2016.

4. M. Džamonja, J. Larson, and W. J. Mitchell, A partition theorem for a large dense linear order, Israel Journal of Mathematics 171 (2009), 237-284.

5. __ Partitions of large Rado graphs, Archive for Mathematical Logic 48 (2009), no. 6, 579-606.

6. A. Hajnal and Komjáth, A strongly non-Ramsey order type, Combinatorica 17 (1997), no. 3.

7. J. D. Halpern and H Läuchli, A partition theorem, Transactions of the American Mathematical Society 124 (1966), 360-367.

8. J. D. Halpern and A. Lévy, The Boolean prime ideal theorem does not imply the axiom of choice, Axiomatic Set Theory, Proc. Sympos. Pure Math., Vol. XIII, Part I, Univ. California, Los Angeles, Calif., 1967, American Mathematical Society, 1971, pp. 83-134.

9. Joel David Hamkins, Small forcing makes any cardinal superdestructible, Journal of Symbolic Logic 63 (1998), 51-58.

10. Claude Laflamme, Norbert Sauer, and Vojkan Vuksanovic, Canonical partitions of universal structures, Combinatorica 26 (2006), no. 2, 183-205.

11. Richard Laver, Products of infinitely many perfect trees, Journal of the London Mathematical Society (2) 29 (1984), no. 3, 385-396.

12. Keith R. Milliken, A Ramsey theorem for trees, Journal of Combinatorial Theory, Series A 26 (1979), 215-237.

13. Norbert Sauer, Coloring subgraphs of the Rado graph, Combinatorica 26 (2006), no. 2, 231-253.

14. Saharon Shelah, Strong partition relations below the power set: consistencywas Sierpinski right? ii, Sets, Graphs and Numbers (Budapest, 1991), vol. 60, Colloq. Math. Soc. János Bolyai, North-Holland, 1991, pp. 637-688.

15. Stevo Todorcevic, Introduction to Ramsey Spaces, Princeton University Press, 2010.

16. Stevo Todorcevic and Ilijas Farah, Some Applications of the Method of Forcing, Yenisei Series in Pure and Applied Mathematics, 1995.

17. Jing Zhang, A tail cone version of the Halpern-Läuchli Theorem at a large cardinal. The Journal of Symbolic Logic, 1-23. doi:10.1017/jsl.2017.55.

Department of Mathematics, University of Denver, C.M. Knudson

Hall, Room 300, 2390 S. York St., Denver, CO 80208 U.S.A.

E-mail address: natasha.dobrinen@du.edu

$U R L:$ http://web.cs.du.edu/ ndobrine

Department of Mathematics, University of Vermont, 16 Colchester

Ave., Burlington, VT 05401 U.S.A.

E-mail address: Daniel.Hathaway@uvm.edu

$U R L:$ http://mysite.du.edu/ dhathaw2/ 